\title{
A Case of Dermatomyositis with Esophageal Fistula in Whom Blind Mucosal Biopsy Detected Occult Oropharyngeal Carcinoma
}

\author{
Miho Kabuto $^{a} \quad$ Noriki Fujimoto $^{a} \quad$ Kazuya Teramura $^{\mathrm{a}}$ Midori Tateishi $^{\mathrm{b}}$ \\ Yasuhito Hamaguchi ${ }^{c}$ Manabu Fujimoto ${ }^{d}$ Toshihiro Tanaka ${ }^{a}$ \\ Departments of a Dermatology and ${ }^{b}$ Otorhinolaryngology, Shiga University of Medical \\ Science, Otsu, 'Department of Dermatology, Graduate School of Medical Science, \\ Kanazawa University, Kanazawa, and ${ }^{\mathrm{d} D e p a r t m e n t}$ of Dermatology, Faculty of Medicine, \\ University of Tsukuba, Tsukuba, Japan
}

\section{Key Words}

Blind biopsy · Dermatomyositis - Esophageal fistula · Oropharyngeal carcinoma · Anti-transcription intermediary factor $1 \cdot$ Truncal erythema

\begin{abstract}
We present a case of anti-transcription intermediary factor 1 (anti-TIF-1) antibody-positive dermatomyositis with concomitant esophageal fistula and extensive truncal erythema. The characteristic cutaneous features and presence of anti-TIF- 1 antibodies were predictive for internal malignancy. However, repeated examinations for internal malignancy showed none, and blind mucosal biopsy was needed to diagnose oropharyngeal carcinoma. We should note the possibility of occult nasopharyngeal carcinoma and consider performing blind mucosal biopsy in dermatomyositis with esophageal fistula, especially with extensive truncal erythema.

(c) 2014 S. Karger AG, Basel
\end{abstract}

\section{Introduction}

Dermatomyositis (DM) and polymyositis (PM) are inflammatory disorders that mainly affect the skin and muscles. Both are often associated with interstitial lung disease and internal malignancy, which considerably determine the prognosis of patients [1]. An association between internal malignancy and inflammatory myopathies has been already well doc- 
umented, particularly in DM [2,3]. Recent reports have revealed that internal malignancy is found in 18-32\% of patients with DM [4]. The diagnosis of occult cancer as early as possible, which can be difficult sometimes, is a very important factor for the prognosis of DM. We present a case of DM with esophageal fistula in whom blind mucosal biopsy was needed to diagnose oropharyngeal carcinoma.

\section{Case Report}

A 65-year-old Japanese male visited our hospital in August 2010. The physical findings were heliotrope rash with periorbital edema, Gottron's sign on the dorsum of the hands (fig. 1) and erythema of the upper arms. He complained of myalgia and slight muscle weakness. He had a prior history of surgical operation for maxillary cancer 27 years before. Laboratory examinations showed almost normal values for complete blood cell counts but elevated levels of aspartate aminotransferase (185 U/l), alanine aminotransferase (106 U/l), lactate dehydrogenase $(637 \mathrm{U} / \mathrm{l}), \mathrm{C}$-reactive protein $(0.5 \mathrm{mg} / \mathrm{dl})$ and creatine kinase $(5,518$ $\mathrm{U} / \mathrm{l}$ ). The anti-nuclear antibody titer was 1:40, and autoantibodies, including anti-Jo-1, antiss-DNA, anti-ds-DNA, anti-Sm, anti-Ro (SS-A), anti-La (SS-B) and anti-RNP antibodies were not detected using enzyme-linked immunosorbent assay. Immunoprecipitation assays revealed the presence of anti-transcription intermediary factor 1 (anti-TIF-1) antibody. Histopathological examination of a biopsy specimen taken from the Gottron's lesion revealed vacuolar degeneration of the basement membrane zone and perivascular lymphocyte infiltration (fig. 2). A deltoid muscle biopsy showed degenerated muscle fiber and infiltration of mononuclear cells. Electromyography showed no myogenic change. Screening for internal malignancy using upper and lower gastrointestinal endoscopy and total body computed tomography (CT) scan showed no abnormality, especially no malignancy or interstitial lung disease. We diagnosed the patient with DM and initiated treatment with prednisolone at $80 \mathrm{mg} /$ day. Blood chemistry examination and symptoms improved; hence, the dosage of prednisolone was gradually reduced.

In December 2010, the patient began to experience dyspnea. Laryngoscopy showed edema of the left pharynx, pressed larynx and narrowed glottides. A neck and chest CT scan demonstrated posterior neck abscess and massive free air from the submandibular to superior mediastinum areas without any sign of malignancy. Upper gastrointestinal endoscopy revealed posterior esophageal fistula. Antibiotic treatment for about 3 weeks led to improvement of the posterior neck abscess and esophageal fistula without surgery.

In May 2011, widespread erythema over the upper body was observed (fig. 3), and laboratory examination revealed an elevated serum creatine kinase level of 1,517 U/l. Although we increased the dosage of prednisolone from 10 to $15 \mathrm{mg} /$ day, the serum creatine kinase level did not decrease. Therefore, we added intravenous immunoglobulin therapy. Repeated examination for internal malignancy with CT scan, gallium scintigraphy and upper and lower gastrointestinal endoscopy showed no recurrence of neck abscess or malignancy, except for intraepithelial neoplasia of the esophagus. Intravenous immunoglobulin therapy did not lead to complete remission of the serum muscle enzyme levels and skin erythema.

In August 2011, the creatine kinase level was elevated again (629 U/l) with concomitant dysphagia. Total body CT scan showed swelling of the right cervical lymph nodes and multiple nodules in both the lungs and liver. Histopathological examination of a biopsy specimen taken from a cervical lymph node presented poorly differentiated squamous cell carcinoma. Since the original lesion of the carcinoma was unidentified, fluorine-18-fluorodeoxyglucose positron emission tomography (FDG PET)/CT scan was performed which revealed uptake in 
the pharynx. Laryngoscopy still showed no abnormality or sign of malignancy in the pharynx. However, blind biopsy from normal-looking pharyngeal mucosa was performed (fig. 4), which revealed poorly differentiated oropharyngeal carcinoma with similar histopathology to the cervical lymph node biopsy. The patient died from metastasis of the oropharyngeal carcinoma a few months later in spite of chemotherapy.

\section{Discussion}

Although cancer screening is usually recommended in patients with DM, there is no consensus regarding how often the screening should be conducted, and it has not been applied systematically to the diagnosis of occult cancer [5]. The sensitivity and specificity of FDG PET/CT for detecting occult cancer in DM are similar compared to those of conventional examination, including CT scan, mammography, gynecologic examination, ultrasonography and tumor marker analysis. Moreover, false-positive and false-negative results occur with both approaches, and a combination of these approaches does not significantly increase the predictive value regarding cancer associated with DM/PM [5]. Nasopharyngeal carcinoma associated with DM is a rare but specific entity [4]. It has been said that blind biopsy should be performed in patients with cervical carcinoma of unknown primary source since oropharyngeal tumors sometimes grow submucosally [6]. Since autopsy was not performed in our case, the exact reason for esophageal fistula was unclear; however, we speculate that oropharyngeal carcinoma was associated with the esophageal fistula.

Recently described anti-TIF-1 autoantibodies against a 155-kDa protein and a 155/ 140-kDa doublet, which are considered to be the same antibodies, have been shown to be significantly associated with cancer in adult patients with DM [7]. The incidence of cancer in anti-TIF-1-positive patients with adult DM is reported to be as high as $42-75 \%$ [8]. The TIF-1 family is a subgroup of the tripartite motif-containing proteins and consists of at least three members, namely TIF- $1 \alpha$, TIF- $1 \beta$ and TIF- $1 \gamma$ [8]. The 155 and $140-\mathrm{kDa}$ proteins recognized by anti-155/140 antibodies are TIF- $1 \gamma$ and TIF- $1 \alpha$, respectively. Anti-155/140 antibodies react with both TIF- $1 \alpha$ and TIF- $1 \gamma$ or only TIF- $1 \gamma$ but not with TIF- $1 \alpha$ alone.

Anti-TIF-1 antibodies might serve as a diagnostic serological marker for cancer associated with DM, occult cancer in particular [1]. Since the analysis of anti-TIF-1 antibodies is not always possible in all institutes, we still need to predict malignancy based on clinical features at an early stage. However, there are few reports that evaluate the cutaneous features of DM associated with the presence of anti-TIF- 1 antibodies. Both the incidence of malignancy and the presence of truncal erythema were observed to be significantly higher in patients with anti-TIF- $1 \alpha$ and anti-TIF- $1 \gamma$ antibodies compared with patients with antiTIF- $1 \gamma$ antibodies alone [8]. Long before the identification of anti-TIF-1 antibodies it was already reported that extensive cutaneous truncal lesions suggest a high mortality rate of DM [9]. The extensive cutaneous truncal lesions seem to be similar to the eruptions referred to as truncal erythema, which corresponds to widespread erythema over the upper body observed in our case. In another report, heliotrope rash, Gottron's sign and flagellate erythema were said to be significantly more frequent in anti-TIF-1 antibody-positive DM patients compared with anti-TIF-1 antibody-negative DM patients [1]. However, extensive cutaneous truncal lesion was not evaluated in the said report, and the eruption might be associated with the presence of anti-TIF-1 antibodies. A characteristic clinical feature despite the skin manifestations of anti-TIF-1 antibody-positive DM patients includes the complete absence of interstitial lung disease. 


\begin{tabular}{l|l}
\hline DOI: $10.1159 / 000368274$ & $\begin{array}{l}\text { C 2014 S. Karger AG, Basel } \\
\text { www.karger.com/cde }\end{array}$ \\
\hline
\end{tabular}

Kabuto et al.: A Case of Dermatomyositis with Esophageal Fistula in Whom Blind Mucosal Biopsy Detected Occult Oropharyngeal Carcinoma

In our case, heliotrope rash, Gottron's sign and widespread erythema over the upper body were observed, and interstitial lung disease was absent. Moreover, anti-TIF-1 antibodies were detected. Based on these observations, the presence of malignancy was predicted. Therefore, repeated examinations for internal malignancy, including upper and lower gastrointestinal endoscopy, CT scan, gallium scintigraphy and laryngoscopy, were performed and showed no abnormality. Even when CT scan detected neoplastic swelling of the cervical lymph nodes and FDG PET/CT showed abnormal uptake in the pharynx, laryngoscopy did not show any signs of malignancy. Blind mucosal biopsy was needed for the definitive diagnosis of oropharyngeal carcinoma. We should note the possibility of occult nasopharyngeal carcinoma and consider performing blind mucosal biopsy in DM patients with posterior esophageal fistula, especially with extensive truncal erythema like in our case.

\section{Disclosure Statement}

The authors report no conflict of interest.

\section{References}

-1 Kaji K, Fujimoto M, Hasegawa M, Kondo M, Saito Y, Komura K, Matsushita T, Orito H, Hamaguchi Y, Yanaba K, Itoh M, Asano Y, Seishima M, Ogawa F, Sato S, Takehara K: Identification of a novel autoantibody reactive with 155 and $140 \mathrm{kDa}$ nuclear proteins in patients with dermatomyositis: an association with malignancy. Rheumatology (Oxford) 2007;46:25-28.

-2 Buchbinder R, Forbes A, Hall S, Dennett X, Giles G: Incidence of malignant disease in biopsy-proven inflammatory myopathy. A population-based cohort study. Ann Intern Med 2001;134:1087-1095.

-3 Sigurgeirsson B, Lindelof B, Edhag 0, Allander E: Risk of cancer in patients with dermatomyositis or polymyositis. A population-based study. N Engl J Med 1992;326:363-367.

-4 Chakroun A, Guigay J, Lusinchi A, Marandas P, Janot F, Hartl DM: Paraneoplastic dermatomyositis accompanying nasopharyngeal carcinoma: diagnosis, treatment and prognosis. Eur Ann Otorhinolaryngol Head Neck Dis 2011;128:127-131.

-5 Selva-O'Callaghan A, Grau JM, Gámez-Cenzano C, Vidaller-Palacín A, Martínez-Gómez X, Trallero-Araguás E, Andía-Navarro E, Vilardell-Tarrés M: Conventional cancer screening versus PET/CT in dermatomyositis/ polymyositis. Am J Med 2010;123:558-562.

6 Haas I, Hoffmann TK, Engers R, Ganzer U: Diagnostic strategies in cervical carcinoma of an unknown primary (CUP). Eur Arch Otorhinolaryngol 2002;259:325-333.

-7 Trallero-Araguás E, Rodrigo-Pendás JÁ, Selva-O'Callaghan A, Martínez-Gómez X, Bosch X, Labrador-Horrillo M, Grau-Junyent JM, Vilardell-Tarrés M: Usefulness of anti-p155 autoantibody for diagnosing cancerassociated dermatomyositis: a systematic review and meta-analysis. Arthritis Rheum 2012;64:523-532.

-8 Fujimoto M, Hamaguchi Y, Kaji K, Matsushita T, Ichimura Y, Kodera M, Ishiguro N, Ueda-Hayakawa I, Asano Y, Ogawa F, Fujikawa K, Miyagi T, Mabuchi E, Hirose K, Akimoto N, Hatta N, Tsutsui K, Higashi A, Igarashi A, Seishima M, Hasegawa M, Takehara K: Myositis-specific anti-155/140 autoantibodies target transcription intermediary factor 1 family proteins. Arthritis Rheum 2012;64:513-522.

-9 Basset-Seguin N, Roujeau JC, Gherardi R, Guillaume JC, Revuz J, Touraine R: Prognostic factors and predictive signs of malignancy in adult dermatomyositis. A study of 32 cases. Arch Dermatol 1990;126: 633-637. 


\section{Case Reports in Dermatology}

Case Rep Dermatol 2014;6:268-273

DOI: $10.1159 / 000368274$

Kabuto et al:: A Case of Dermatomyositis with Esophageal Fistula in Whom Blind Mucosal Biopsy Detected Occult Oropharyngeal Carcinoma

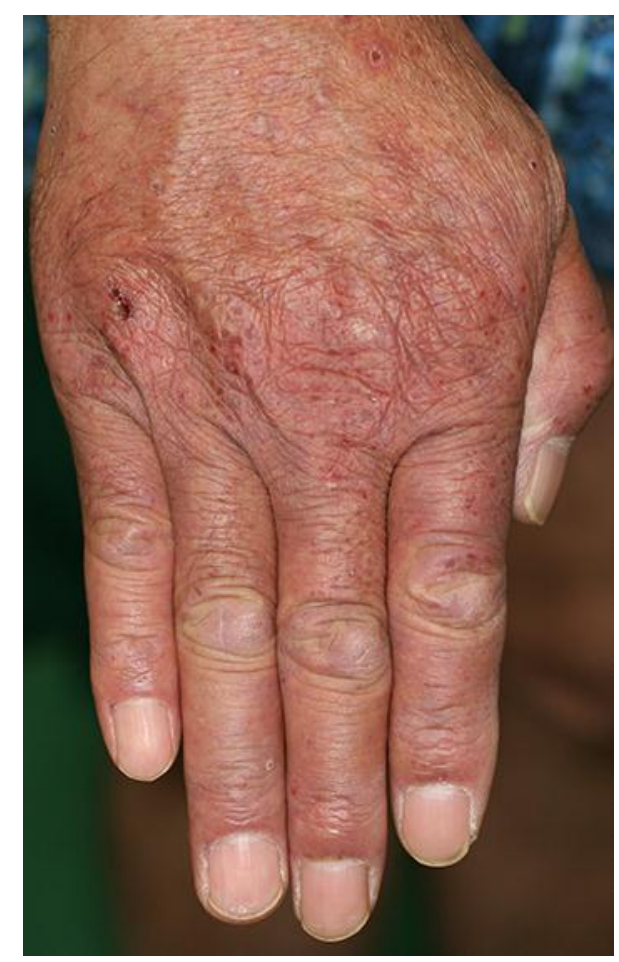

Fig. 1. Clinical presentation. Gottron's sign on the dorsum of the hands were observed at initial visit.

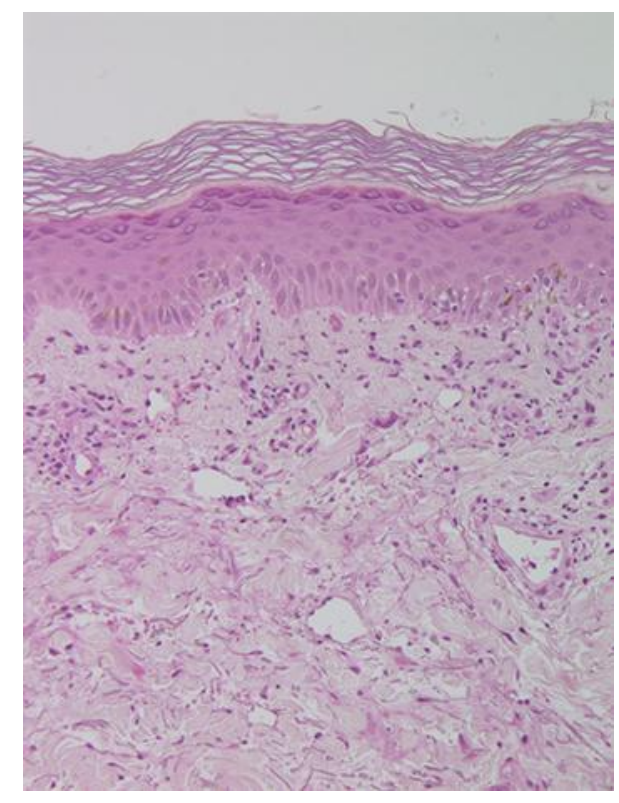

Fig. 2. Histopathological findings in a skin biopsy specimen taken from the Gottron's lesion. Vacuolar degeneration of the basement membrane zone and perivascular lymphocyte infiltration were observed (hematoxylin \& eosin staining, original magnification $\times 100$ ). 
Kabuto et al.: A Case of Dermatomyositis with Esophageal Fistula in Whom Blind Mucosal Biopsy Detected Occult Oropharyngeal Carcinoma

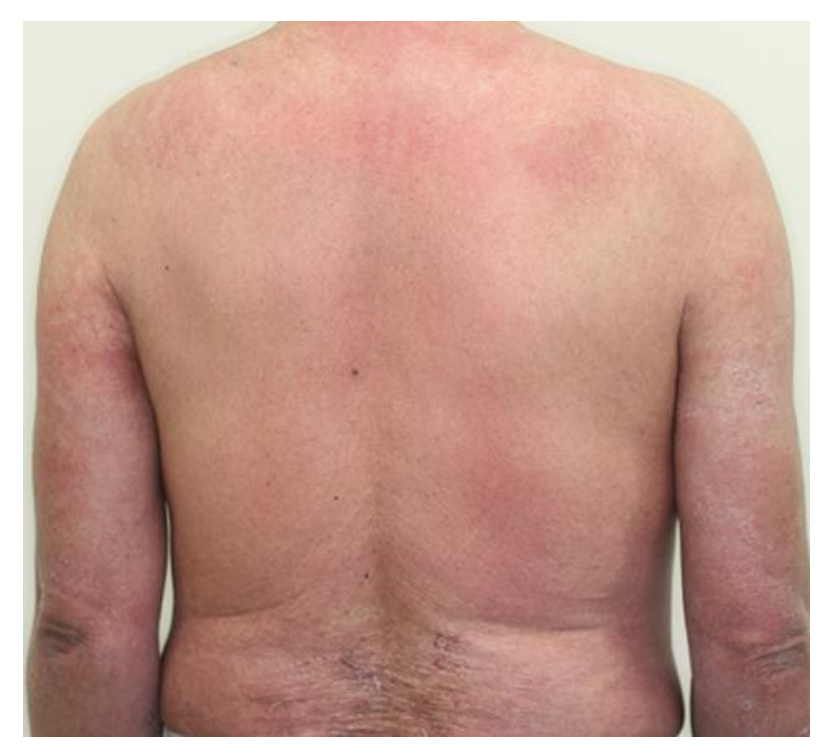

Fig. 3. Clinical presentation. Widespread erythema all over the upper body was observed in May 2011.

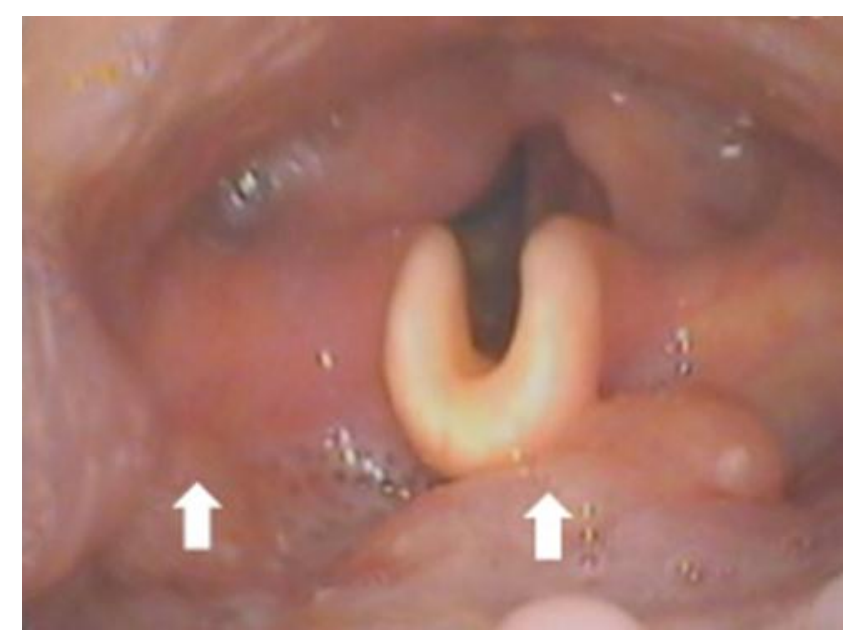

Fig. 4. Laryngoscopic finding. No abnormality or sign of malignancy was observed in the pharynx. The white arrows show sites in which poorly differentiated oropharyngeal carcinoma was revealed by blind mucosal biopsy. 